\section{Assessing Mitral Valve Stenosis by Real-time 3-Dimensional Echocardiography in Systemic Lupus Erythematosus: A Look Inside the Heart}

To the Editor:

Heart valve pathology is commonly recognized in systemic lupus erythematosus (SLE) ${ }^{1}$. Immunoglobulin and complement deposition in the valvular structure consequently leads to valve thickening, Libman-Sacks vegetations, and valve regurgitation ${ }^{1}$. Involvement of the mitral valve (MV) is most frequently encountered. Valvular stenosis is rarely seen ${ }^{1}$. This is the first report to describe the value of live real-time 3-dimensional echocardiography (RT3DE) in the diagnosis of SLE-related MV stenosis.

A 68-year-old woman with a history of longstanding SLE and new onset of atrial fibrillation was admitted with dyspnea on exertion over the past year, deteriorating progressively during the last month. A standard transthoracic 2-dimensional (2-D) echocardiogram performed with a Vivid 7 Dimension system (GE Medical Systems, Milwaukee, WI, USA) revealed normal left ventricular size and systolic function, right ventricular dilatation with impaired systolic function, left atrium enlargement, MV leaflet thickening, and mild MV stenosis (MV area $=1.6 \mathrm{~cm}^{2}$, Figure $1 \mathrm{~A}$ ) with trivial regurgitation. Moderate tricuspid regurgitation was detected with estimated right ventricular systolic pressure of approximately $60 \mathrm{~mm}$ $\mathrm{Hg}$. After the 2-D examination, complementary MV assessment by RT3DE was carried out, using the same ultrasound system and a $3 \mathrm{~V}$ full-matrix array transducer capable of online, real-time non-gated 3-D imaging and rendering as well as full-volume 3-D imaging with gated acquisition. The resultant 3-D data were processed using dedicated software (EchoPAC; GE Medical Systems). RT3DE confirmed the 2-D echocardiographic findings with respect to MV morphology and severity of stenosis (Figure 1B, 1C, 1D).

Comparing with 2-D echocardiography, RT3DE allows a more "realistic" visualization of the heart ${ }^{2}$. Implementation of the RT3DE with the new generation of full-matrix array transducers, capable of online 3-D rendering, offers advantages over 2-D echocardiography for assessment of the morphology, function, and pathology of the MV apparatus. Due to the variable geometry of the stenotic MV orifice, correct plane orientation frequently becomes difficult. Navigation and cropping tools incorporated into the commercial software packages for 3-D data analysis allow optimization of the cut planes that would provide unique en face views of the stenotic MV from which accurate measurements of the MV area can be made (Figure 1D). Available evidence suggests that RT3DE provides improved accuracy and reproducibility over 2-D methods for the calculation of MV area in patients with rheumatic and calcific MV stenosis ${ }^{2,3}$, yet its use in SLE-related valvulopathy has not been clinically implemented. In patients with SLE and suspected cardioembolism, acute or subacute Libman-Sacks endocarditis with moderate or worse valve dysfunction, transesophageal 2-D echocardiography appears superior to transthoracic 2-D echocardiography $^{4}$. The diagnostic accuracy of transesophageal 2-D echocardiography in comparison with that of transthoracic RT3DE, in this group of patients, remains to be examined.

SOTIRIS C. PLASTIRAS, MD, Research Fellow in Cardiology, Department of Clinical Therapeutics, Echocardiography Unit, University of Athens Medical School, Alexandra Hospital, Department of Pathophysiology, University of Athens Medical School, Laiko Hospital; CONSTANTINOS A. PAMBOUCAS, MD, Consultant of Cardiology, Department of Clinical Therapeutics, Echocardiography Unit, University of Athens Medical School, Alexandra Hospital; GEORGE E. TZELEPIS, MD, Associate Professor of Medicine, Department of Pathophysiology, University of Athens Medical School, Laiko Hospital; SAVVAS T. TOUMANIDIS, MD, Associate Professor of Cardiology, Department of Clinical Therapeutics, Echocardiography Unit, University of Athens Medical School, Alexandra Hospital, Athens, Greece. Address reprint requests to Dr. S.C. Plastiras, Echocardiography Unit, University of Athens Medical School, Alexandra Hospital, 80 Vasilisis Sofias Ave and Lourou St., 11528 Athens, Greece. E-mail: splastiras@panafonet.gr

We thank Prof. Haralampos Moutsopoulos for his continuous inspiration, guidance, and support.

\section{REFERENCES}

1. Moyssakis I, Tektonidou MG, Vasilliou VA, et al. Libman-Sacks endocarditis in systemic lupus erythematosus: prevalence, associations, and evolution. Am J Med 2007;120:636-42.

2. Chu JW, Levine RA, Chua S, et al. Assessing mitral valve area and orifice geometry in calcific mitral stenosis: a new solution by real-time three-dimensional echocardiography. J Am Soc Echocardiogr 2008;21:1006-9.

3. Zamorano J, Cordeiro P, Sugeng L, et al. Real-time three-dimensional echocardiography for rheumatic mitral valve stenosis evaluation: an accurate and novel approach. J Am Coll Cardiol 2004;43:2091-6

4. Roldan CA, Qualls CR, Sopko KS, et al. Transthoracic versus transesophageal echocardiography for detection of Libman-Sacks endocarditis: a randomized controlled study. J Rheumatol 2008;35:224-9.

J Rheumatol 2009;36:8; doi:10.3899/jrheum.090039 

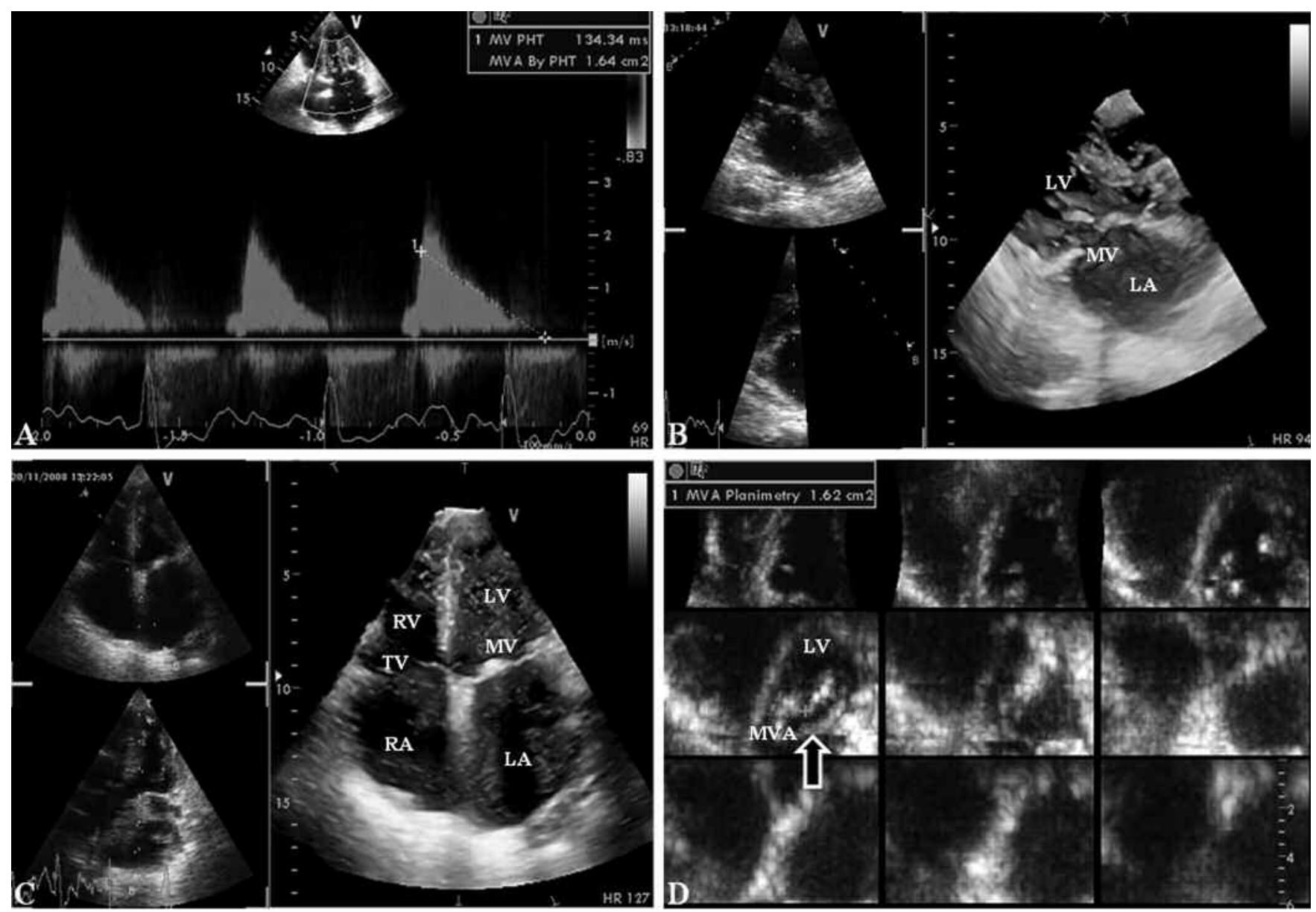

Figure 1. A. Pressure half time measurement from continuous-wave Doppler velocity recordings across the mitral valve (MV). The PHT of $134 \mathrm{~ms}$ corresponds to a valve area of $1.6 \mathrm{~cm}^{2}$. B. Parasternal long-axis view of the stenotic MV using real-time 3-D echocardiography. With this mode, predominantly used to visualize cardiac valve morphology, a 3-D pyramidal data set of approximately $50^{\circ} \times 30^{\circ}$ is displayed in a volume-rendered manner in real time without the need for respiratory gating. MV leaflet thickening is clearly visible. C. Wide-angle acquisition from the apical 4-chamber view providing a pyramidal data set of approximately $90^{\circ} \times 90^{\circ}$, which allows inclusion of a larger cardiac volume. In this mode, the wide-angle data set is compiled by merging 4 narrower wedges obtained over 4 consecutive cardiac cycles during a breath-hold with electrocardiograph gating. D. MV area measurement by 3-D echocardiography using a novel cropping tool, the 9-slice mode. The wide-angle pyramidal data set obtained from a typical apical 4-chamber view is sliced into 9 equidistant, parallel short-axis cut planes from base to apex. The position of the 9 slices can be adapted to the real anatomy in angle and distance. Thus, it is possible to pinpoint the cut plane to the tips of the MV so that the true anatomic valve area can be measured by planimetry. RT3DE-derived measurement $\left(1.62 \mathrm{~cm}^{2}\right)$ showed an almost identical agreement with that obtained by the pressure half time method. PHT: pressure half time; LV: left ventricle; LA: left atrium; MV: mitral valve; RA: right atrium; RV: right ventricle; TV: tricuspid valve; ECG: electrocardiogram; RT3DE: real-time 3-D echocardiography. 